\title{
The Numerical Evaluation of Very Oscillatory Infinite Integrals by Extrapolation
}

\author{
By Avram Sidi
}

\begin{abstract}
Recently the author has given two modifications of a nonlinear extrapolation method due to Levin and Sidi, which enable one to accurately and economically compute certain infinite integrals whose integrands have a simple oscillatory behavior at infinity. In this work these modifications are extended to cover the case of very oscillatory infinite integrals whose integrands have a complicated and increasingly rapid oscillatory behavior at infinity. The new method is applied to a number of complicated integrals, among them the solution to a problem in viscoelasticity. Some convergence results for this method are presented.
\end{abstract}

1. Introduction. Recently Levin and Sidi [4] have given some nonlinear transformations for accelerating the convergence of slowly converging infinite integrals and series, namely the $D$ - and $d$-transformations, respectively, which have proved to be very efficient numerically. Convergence properties of these transformations, which are generalizations of the Richardson extrapolation process, have been analyzed in a series of papers by the present author, and some results, which, to a certain extent, explain how these transformations work, have been given, see Sidi [9], [10], [11]. Actually, Sidi [9], [11] deal specifically with the case of the $T$-transformation of Levin [2], which is a special case of the $d$-transformation, and Sidi [10] deals with the generalized Richardson extrapolation process, which has as special cases the $D$ - and $d$-transformations.

Two useful modifications of the $D$-transformation (the $\bar{D}$ - and $\tilde{D}$-transformations) that simplify the computation of oscillatory infinite integrals, with special emphasis on Fourier and Hankel transforms, have been given by the present author, see Sidi [12]. Also for this case convergence results have been proved. The methods developed in [12] make extensive use of the simple oscillatory behavior of the integrand, which is merely a sine or a cosine (or a combination of both) of the integration variable. This is especially so for Fourier and Hankel transforms.

In the present work we give a simple yet efficient procedure, namely the $W$ transformation, for accelerating the convergence of very oscillatory infinite integrals whose integrands have a complicated and increasingly rapid oscillatory behavior at infinity. In the remainder of this section we shall explain in detail what exactly is meant by the phrase "very oscillatory".

Following Levin and Sidi [4], Sidi [10], [12], we give the definition below:

Definition 1.1. We shall say that a function $\alpha(x)$, defined for $x>a \geqslant 0$, belongs to the set $A^{(\gamma)}$, if it is infinitely differentiable for all $x>a$, and if, as $x \rightarrow \infty$, it has a

Received December 6, 1979; revised February 25, 1981.

1980 Mathematics Subject Classification. Primary 41A55, 41A60, 40A25, 40A05.

(C)1982 American Mathematical Society $0025-5718 / 81 / 0000-0086 / \$ 04.00$ 
Poincare-type asymptotic expansion of the form

$$
\alpha(x) \sim x^{\gamma} \sum_{i=0}^{\infty} \alpha_{i} / x^{i},
$$

and all its derivatives, as $x \rightarrow \infty$, have Poincaré-type asymptotic expansions, which are obtained by differentiating the right-hand side of (1.1) term by term.

Remark 1. From this definition it follows that $A^{(\gamma)} \supset A^{(\gamma-1)} \supset A^{(\gamma-2)} \supset \ldots$.

Remark 2. It can easily be verified that if $\alpha \in A^{(\gamma)}$ and $\beta \in A^{(\delta)}$, then $\alpha \beta \in A^{(\gamma+\delta)}$, and if, in addition, $\beta \notin A^{(\delta-1)}$, then $\alpha / \beta \in A^{(\gamma-\delta)}$.

Remark 3. If $\alpha \in A^{(0)}$, then it is infinitely differentiable for all $x>a$ up to and including $x=\infty$, although not necessarily analytic at $x=\infty$.

The integrals treated in this work are of the form:

$$
I[f]=\int_{a}^{\infty} f(t) d t \text { for some } a \geqslant 0,
$$

where the integrand $f(x)$ can be expressed as

$$
f(x)=u(\theta(x)) e^{\phi(x)} h(x),
$$

where

(1) $u(t)$ denotes either $\cos t$ or $\sin t$,

(2) $\theta(x)$ is real and $\theta \in A^{(m)}$ for some positive integer $m$,

(3) $\phi(x)$ is real and $\phi \in A^{(k)}$ for some nonnegative integer $k$, and $\lim _{x \rightarrow \infty} \phi(x)=$ $-\infty$ if $k \geqslant 1$,

(4) $h(x)$ is real and $h \in A^{(\gamma)}$ for some $\gamma$, such that $f(x)$ is integrable at $x=\infty$. A simple analysis shows that when $k \geqslant 1, f(x)$ is integrable at $x=\infty$ for any value of $\gamma$, and when $k=0, f(x)$ is integrable at $x=\infty$ provided $\gamma<m-1$.

Example 1.1.

$$
\begin{aligned}
f(x)= & \sin \left(x^{3}+2 x+4+\frac{x}{x^{2}+3}\right) \\
& \times \exp \left(-x^{2}+3 x+2+\sqrt{x^{2}+x / 2+1}\right) \frac{x^{5}+x^{2}}{\sqrt{x^{3}+x^{2}}} .
\end{aligned}
$$

Here $m=3, k=2, \gamma=7 / 2$.

If $m=1$, then $f(x)$ oscillates at infinity like $\sin c x$ or $\cos c x$ where $c$ is a constant. This is the case treated in Sidi [12]. If $m \geqslant 2$, however, $f(x)$ oscillates very rapidly at infinity, in the sense that as $\xi \rightarrow \infty$, the number of times $f(x)$ changes its sign in the interval $[\xi, \xi+\Delta \xi]$, with $\Delta \xi>0$ fixed, also tends to infinity; equivalently the distance between two consecutive zeros of $f(x)$ tends to zero as these zeros approach infinity.

Let us define

$$
F(x)=\int_{a}^{x} f(t) d t
$$

With the help of a theorem due to Levin and Sidi [4], in Section 2, we obtain an asymptotic expansion for $I[f]-F(x)$ as $x \rightarrow \infty$. Through this asymptotic expansion, in Section 3, the $W$-transformation is defined as an extrapolation process based on the $F\left(x_{l}\right)$, for a small number of carefully chosen values of $x_{l}$. In Section 4 we 
illustrate the $W$-transformation with several numerical examples. In Section 5 we supply some results on the convergence properties of the $W$-transformation.

2. Theory. Let the function $g(x)$ be expressible in the form

$$
g(x)=e^{i \theta(x)} e^{\phi(x)} h(x)
$$

where $\theta(x), \phi(x)$, and $h(x)$ are exactly as in the previous section with the same notation. (From (2.1) it follows that the function $f(x)$ given in (1.3) is simply the real or the imaginary part of $g(x)$.) Let us define

$$
I[g]=\int_{a}^{\infty} g(t) d t \text { and } G(x)=\int_{a}^{x} g(t) d t
$$

as in (1.2) and (1.4). Our purpose now is to obtain an asymptotic expansion for

$$
I[g]-G(x)=\int_{x}^{\infty} g(t) d t
$$

in the limit $x \rightarrow \infty$. For this we need the following result, which is a special case of a theorem due to Levin and Sidi [4].

THEOREM 2.1. Let $v(x)$ be defined for $x>a \geqslant 0$, and be integrable at $x=\infty$, and satisfy a linear first-order homogeneous differential equation of the form

$$
v(x)=p(x) v^{\prime}(x),
$$

where $p \in A^{(r)}$ but $p \notin A^{(r-1)}$, such that $r$ is an integer less than or equal to 1 . Let also

$$
\lim _{x \rightarrow \infty} p(x) v(x)=0 \text {. }
$$

If for the integers $l=-1,1,2,3, \ldots$,

$$
\bar{p} l \neq 1
$$

where

$$
\bar{p}=\lim _{x \rightarrow \infty} x^{-1} p(x)
$$

then

$$
\int_{x}^{\infty} v(t) d t=x^{r} v(x) \beta(x)
$$

such that $\beta \in A^{(0)}$. (In the original result of Levin and Sidi [4] the right-hand side of (2.7) is given as $x^{\tau} v(x) \beta(x)$ with $\tau$ being an integer less than or equal to $r$. But for $v(x)$ as above, going through the steps of the proof of the theorem of Levin and Sidi [4], one can easily see that $\tau=r$ exactly.)

In the appendix at the end of the present work we shall present a new approach to the derivation of $\beta(x)$ from a differential equation, which is different than that given in the work of Levin and Sidi.

We now apply Theorem 2.1 to the function $g(x)$.

THEOREM 2.2. Let $g(x)$ be as defined in the beginning of this section. Then

$$
\int_{x}^{\infty} g(t) d t=x^{\sigma} g(x) \beta(x)
$$

where

$$
\sigma=\min \{-m+1,-k+1\}
$$

and $\beta \in A^{(0)}$. 
Proof. We shall show that all the conditions of Theorem 2.1 are satisfied by the function $g(x)$. First of all $g(x)$ satisfies the linear first-order homogeneous differential equation $g(x)=s(x) g^{\prime}(x)$, where

$$
s(x)=\left[i \theta^{\prime}(x)+\phi^{\prime}(x)+h^{\prime}(x) / h(x)\right]^{-1} .
$$

Since $\theta^{\prime} \in A^{(m-1)}, \phi^{\prime} \in A^{(k-1)},\left(h^{\prime} / h\right) \in A^{(-1)}$, and $m \geqslant 1, k \geqslant 0$, we see that

$$
1 / s=\left(i \theta^{\prime}+\phi^{\prime}+h^{\prime} / h\right) \in A^{(\rho)},
$$

but $(1 / s) \notin A^{(\rho-1)}$, where $\rho=\max \{m-1, k-1\}$. By Remark 2 in Section 1, we then see that $s \in A^{(\sigma)}$ but $s \notin A^{(\sigma-1)}$. Since $\gamma<m-1$ when $k=0$ and arbitrary otherwise, and $s \in A^{(\sigma)}$, we can easily see that $\lim _{x \rightarrow \infty} s(x) g(x)=0$, hence (2.4) is satisfied. (2.5) is satisfied trivially because $\bar{s}=\lim _{x \rightarrow \infty} x^{-1} s(x)=0$ by the fact that $s \in A^{(\sigma)}$ and $\sigma \leqslant 0$. Hence, Theorem 2.1 applies to $g(x)$, consequently (2.8) holds with $\beta \in A^{(0)}$. This completes the proof of the theorem.

Now $\theta(x)$, being in $A^{(m)}$, has an asymptotic expansion of the form

$$
\theta(x) \sim x^{m} \sum_{i=0}^{\infty} \theta_{i} / x^{i} \text { as } x \rightarrow \infty .
$$

Hence $\theta(x)$ is of the form $\theta(x)=\bar{\theta}(x)+\Delta(x)$, where $\bar{\theta}(x)$ is a polynomial of degree $m$, and $\Delta(x)$ is in $A^{(0)}$. Specifically,

$$
\bar{\theta}(x)=\sum_{i=0}^{m-1} \theta_{i} x^{m-i}, \quad \Delta(x) \sim \sum_{i=0}^{\infty} \theta_{m+i} / x^{i} \quad \text { as } x \rightarrow \infty .
$$

Therefore,

$$
e^{i \theta(x)}=e^{i \bar{\theta}(x)} \delta(x),
$$

where $\delta(x)=e^{i \Delta(x)}$, and $\delta \in A^{(0)}$ since $\Delta \in A^{(0)}$.

Similarly, $\phi \in A^{(k)}$ implies that $\phi(x)=\bar{\phi}(x)+\Lambda(x)$, where $\bar{\phi}(x)$ is a polynomial of degree $k$, and $\Lambda(x)$ is in $A^{(0)}$. Hence

$$
e^{\phi(x)}=e^{\bar{\phi}(x)} \lambda(x),
$$

where $\lambda(x)=e^{\Lambda(x)}$, hence $\lambda \in A^{(0)}$.

Example 2.1. In Example 1.1 in the previous section we have $\bar{\theta}(x)=x^{3}+2 x$ and $\bar{\phi}(x)=-x^{2}+4 x$.

Substituting (2.13) and (2.14) in (2.8), and using the fact that $h \in A^{(\gamma)}$, we obtain the following result.

THEOREM 2.3. $I[g]-G(x)$ can be expressed in the form

$$
I[g]-G(x)=x^{\sigma+\gamma} e^{i \bar{\theta}(x)} e^{\bar{\phi}(x)} \beta^{*}(x),
$$

where $\beta^{*}(x)=x^{-\gamma} h(x) \delta(x) \lambda(x) \beta(x)$, hence $\beta^{*} \in A^{(0)}$.

Proof. We only have to show that $\beta^{*} \in A^{(0)}$. Now since $x^{-\gamma}$ is in $A^{(-\gamma)}$ and $h \in A^{(\gamma)}, x^{-\gamma} h(x)$ is in $A^{(0)}$ by Remark 2 of the previous section. We have seen that $\delta \in A^{(0)}$ and $\lambda \in A^{(0)}$. Again by Remark 2 of the previous section the product of any number of functions in $A^{(0)}$ is a function in $A^{(0)}$, hence the result follows. 
COROLLARY. By taking the real or imaginary part of both sides of (2.15) we obtain

$$
I[f]-F(x)=x^{\sigma+\gamma} e^{\bar{\phi}(x)}\left\{\cos [\bar{\theta}(x)] b_{1}(x)+\sin [\bar{\theta}(x)] b_{2}(x)\right\},
$$

where

$$
\begin{aligned}
& b_{1}(x)= \begin{cases}\operatorname{Re} \beta^{*}(x) & \text { if } f(x)=\operatorname{Re} g(x), \\
\operatorname{Im} \beta^{*}(x) & \text { if } f(x)=\operatorname{Im} g(x),\end{cases} \\
& b_{2}(x)= \begin{cases}-\operatorname{Im} \beta^{*}(x) & \text { if } f(x)=\operatorname{Re} g(x), \\
\operatorname{Re} \beta^{*}(x) & \text { if } f(x)=\operatorname{Im} g(x),\end{cases}
\end{aligned}
$$

hence $b_{1} \in A^{(0)}$ and $b_{2} \in A^{(0)}$.

Remark 1. So far we have assumed that $h(x)$ is a real function. However, the result stated in Theorem 2.3 is valid also when $h(x)$ is complex, since we have not made use of the assumption that $h(x)$ is real, in any of the steps that lead to (2.15). From this it can easily be verified that (2.16) in the corollary to Theorem 2.3 is valid whenever $h(x)$ is complex, with $b_{1} \in A^{(0)}$ and $b_{2} \in A^{(0)}$, the only difference being that $b_{1}(x)$ and $b_{2}(x)$ are not given by (2.17) but by slightly more complicated expressions. In the next lemma we show that $(2.16)$ is valid for functions $f(x)$ with a more complicated appearance than considered so far.

LEMMA 2.1. Let $f(x)$ be of the form

$$
f(x)=\sum_{i=1}^{r} f_{i}(x)
$$

where each of the $f_{i}(x)$ is of the form

$$
f_{i}(x)=u_{i}\left(\theta_{i}(x)\right) e^{\phi_{i}(x)} h_{i}(x), \quad i=1, \ldots, r,
$$

such that

(1) $u_{i}(t)$ is either $\cos t$ or $\sin t$ (or a linear combination of both, like $e^{ \pm i t}$ );

(2) each of the $\theta_{i}(x)$ is a real function in $A^{(m)}, m$ being a positive integer, with the property that $\bar{\theta}_{i}(x) \equiv \bar{\theta}_{j}(x)$ for $i \neq j$;

(3) each of the $\phi_{i}(x)$ is a real function in $A^{(k)}, k$ being a nonnegative integer, with the property that $\bar{\phi}_{i}(x) \equiv \bar{\phi}_{j}(x)$ for $i \neq j$;

(4) each of the $h_{i}(x)$ is a (complex) function in $A^{\left(\gamma_{i}\right)}$, for some $\gamma_{i}$ with the property that $\gamma_{i}-\gamma_{j}=$ integer for $i \neq j$ (hence $h_{i} \in A^{(\gamma)}$ for each $i$, where $\gamma=\max \left\{\gamma_{1}, \ldots, \gamma_{r}\right\}$, see Remark 1 in the previous section).

Then (2.16) holds with $\sigma$ as given in (2.9), $\gamma=\max \left\{\gamma_{1}, \ldots, \gamma_{r}\right\}$, and $b_{1} \in A^{(0)}$ and $b_{2} \in A^{(0)}$.

Proof. The result follows by applying the corollary to Theorem 2.3 to each $f_{i}(x)$, and recalling Remark 1 above. We omit the details.

Example of the Application of Lemma 2.1. Consider

$$
f(x)=\left\{\begin{array}{l}
J_{\nu}(\theta(x)) \\
Y_{\nu}(\theta(x))
\end{array}\right\} e^{\phi(x)} h(x),
$$

where $\theta(x) \geqslant 0, \phi(x)$, and $h(x)$ are as described in the first paragraph of this section and $J_{\nu}(t)$ and $Y_{\nu}(t)$ are the Bessel functions of order $\nu$ of the first and second kind, 
respectively. Let $v(t)$ denote either $J_{\nu}(t)$ or $Y_{\nu}(t)$. Then as $t \rightarrow \infty$

$$
v(t)=\cos t \eta_{1}(t)+\sin t \eta_{2}(t),
$$

where $\eta_{1} \in A^{(-1 / 2)}$ and $\eta_{2} \in A^{(-1 / 2)}$. Since $\theta(x) \rightarrow \infty$ as $x \rightarrow \infty$, we have

$$
\begin{aligned}
v(\theta(x)) & =\cos [\theta(x)] \eta_{1}(\theta(x))+\sin [\theta(x)] \eta_{2}(\theta(x)) \\
& =\cos [\theta(x)] \bar{\eta}_{1}(x)+\sin [\theta(x)] \bar{\eta}_{2}(x),
\end{aligned}
$$

where it can easily be shown that $\bar{\eta}_{1} \in A^{(-m / 2)}$ and $\bar{\eta}_{2} \in A^{(-m / 2)}$. Hence we have shown that $f(x)$, as given in (2.20), can be expressed in the form (2.18) with $r=2$, $u_{1}(t)=\cos t, u_{2}(t)=\sin t, \theta_{1}(x)=\theta_{2}(x)=\theta(x), \phi_{1}(x)=\phi_{2}(x)=\phi(x)$, and $h_{1}(x)$ $=\bar{\eta}_{1}(x) h(x), h_{2}(x)=\bar{\eta}_{2}(x) h(x)$, both being in $A^{(\gamma-m / 2)}$. (We note that these results will be of use in Example 4.4 in Section 4.)

3. The $W$-Transformation. In this section we give an extrapolation method by which approximations to $I[f]$, when $f(x)$ is as in Lemma 2.1, can be obtained as the solution of a system of linear equations.

Let us start with (2.16). Let $x_{0}$ be the smallest zero of $\sin [\bar{\theta}(x)]$ (or of $\cos [\bar{\theta}(x)]$ ) greater than $a \geqslant 0$. Then $x_{0}$ is the solution to the polynomial equation $\bar{\theta}(x)=q \pi$ (or $\bar{\theta}(x)=(q+1 / 2) \pi)$ for some integer $q$. Once $x_{0}$ has been found, we go on to determine $x_{1}<x_{2}<\ldots$, the consecutive zeros of $\sin [\bar{\theta}(x)]$ (or of $\cos [\bar{\theta}(x)]$ ) such that $\cos \left[\bar{\theta}\left(x_{l}\right)\right] \cos \left[\bar{\theta}\left(x_{l+1}\right)\right]<0$ (or $\sin \left[\bar{\theta}\left(x_{l}\right)\right] \sin \left[\bar{\theta}\left(x_{l+1}\right)\right]<0$ ). That is, $x_{l}$ is the solution to the polynomial equation $\bar{\theta}(x)=(q+l) \pi($ or $\bar{\theta}(x)=(q+l+1 / 2) \pi)$. It is clear that $x_{l} \rightarrow \infty$ as $l \rightarrow \infty$; as a matter of fact $x_{l}=O\left(l^{1 / m}\right)$ as $l \rightarrow \infty$.

If we now let $x=x_{l}$ in (2.16), we obtain

$$
I[f]-F\left(x_{l}\right)=\psi\left(x_{l}\right) b\left(x_{l}\right), \quad l=0,1,2, \ldots,
$$

where

$$
\psi(x)= \begin{cases}\cos [\bar{\theta}(x)] \cdot x^{\sigma+\gamma} e^{\bar{\phi}(x)} & \text { if } x_{l} \text { are zeros of } \sin [\bar{\theta}(x)] \\ \sin [\bar{\theta}(x)] \cdot x^{\sigma+\gamma} e^{\bar{\phi}(x)} & \text { if } x_{l} \text { are zeros of } \cos [\bar{\theta}(x)]\end{cases}
$$

and

$$
b(x)= \begin{cases}b_{1}(x) & \text { if } x_{l} \text { are zeros of } \sin [\bar{\theta}(x)] \\ b_{2}(x) & \text { if } x_{l} \text { are zeros of } \sin [\bar{\theta}(x)]\end{cases}
$$

Note that

$$
\psi\left(x_{l}\right)=c(-1)^{l} x_{l}^{\sigma+\gamma} e^{\bar{\phi}\left(x_{l}\right)}, \quad l=0,1,2, \ldots,
$$

where $c=\cos \left[\bar{\theta}\left(x_{0}\right)\right]$ or $c=\sin \left[\bar{\theta}\left(x_{0}\right)\right]$, depending on whether $x_{l}$ are the zeros of $\sin [\bar{\theta}(x)]$ or $\cos [\bar{\theta}(x)]$, respectively. Consequently

$$
\psi\left(x_{l}\right) \psi\left(x_{l+1}\right)<0, \quad l=0,1,2, \ldots .
$$

This is a very important property as will be explained later. 
Definition 3.1 (The W-Transformation). The approximation $W_{n}^{(j)}$ to $I[f]$ and the parameters $\bar{\beta}_{i}, i=0,1, \ldots, n$, are defined to be the solution to the system of $n+2$ linear equations

$$
W_{n}^{(j)}=F\left(x_{l}\right)+\psi\left(x_{l}\right) \sum_{i=0}^{n} \frac{\bar{\beta}_{i}}{x_{l}^{i}}, \quad l=j, j+1, \ldots, j+n+1 .
$$

The inequality in (3.5) guarantees the existence of a unique solution to these equations as has been shown in Sidi [12].

We note that the $W$-transformation is a special case of the generalized Richardson extrapolation process treated by the author in [10].

Previously, the author has considered two kinds of limiting processes, see [9], [10], [11], [12]:

(a) Process $\mathrm{I} ; n$ is fixed, $j \rightarrow \infty$,

(b) Process II, $j$ is fixed, $n \rightarrow \infty$.

The convergence properties of both of these processes is taken up briefly in Section 5. It turns out that Process II has very good convergence properties and is much more efficient than Process I.

A recursive algorithm for the implementation of the $W$-transformation has been developed by Sidi [13], and is denoted as the $W$-algorithm. It turns out that the $W$-algorithm requires very little storage and very few arithmetic operations. Furthermore, it is proved in [13] that whenever (3.5) is satisfied, the $W$-algorithm is stable in that errors in $F\left(x_{l}\right)$ and $\psi\left(x_{l}\right)$ are not magnified. We now describe how the $W$-algorithm can be applied to the $W$-transformation defined by Eqs. (3.6):

(1) Define

$$
\begin{aligned}
& M_{-1}^{(s)}=F\left(x_{s}\right) / \psi\left(x_{s}\right), \quad s=0,1, \ldots \\
& N_{-1}^{(s)}=1 / \psi\left(x_{s}\right),
\end{aligned}
$$

(2) Let

$$
\left\{\begin{array}{l}
M_{k}^{(s)}=\left(M_{k-1}^{(s)}-M_{k-1}^{(s+1)}\right) /\left(x_{s}^{-1}-x_{s+k+1}^{-1}\right), \\
N_{k}^{(s)}=\left(N_{k-1}^{(s)}-N_{k-1}^{(s+1)}\right) /\left(x_{s}^{-1}-x_{s+k+1}^{-1}\right), \quad s=0,1, \ldots, k=0,1, \ldots \\
W_{k}^{(s)}=M_{k}^{(s)} / N_{k}^{(s)}
\end{array}\right.
$$

For details see [13].

4. Numerical Examples. In this section we shall give four numerical examples that show the accuracy of the method presented in the previous section when applied to very oscillatory integrals. All the results have been obtained by using the $W$ transformation of Section 3, for Process II using $j=0$, since Process II is the more efficient of the two processes. (See also Theorem 5.1.)

Example 4.1. $\int_{0}^{\infty} \sin \left(\pi t^{2} / 2\right) / d t=1 / 2$. For this case $u(t)=\sin t, \theta(x)=$ $\bar{\theta}(x)=(\pi / 2) x^{2}, \phi(x)=$ constant, and $\gamma=0$. Hence $x_{l}, l=0,1, \ldots$, are roots of the equation $(\pi / 2) x^{2}=(l+1) \pi, l=0,1, \ldots$, i.e., $x_{l}=\sqrt{2(l+1)}, l=0,1, \ldots$ Since $m=2$ and $k=0$, we have $\sigma=-1$. Therefore, $\psi(x)=\cos \left(\pi x^{2} / 2\right) / x$ and $\psi\left(x_{l}\right)=(-1)^{l+1} / x_{l}, l=0,1, \ldots$ Table 4.1 contains some of the results of the computations for this integral. 
TABLE 4.1

Approximations $W_{n}^{(0)}$ for the integral $\int_{0}^{\infty} \sin \left(\pi t^{2} / 2\right) d t=\frac{1}{2}$.

\begin{tabular}{|r|l|}
\hline$n$ & \multicolumn{1}{|c|}{$W_{n}^{(0)}$} \\
\hline 1 & 0.4997 \\
3 & 0.500002 \\
5 & 0.499999991 \\
7 & 0.50000000004 \\
9 & 0.4999999999998 \\
11 & 0.5000000000000009 \\
\hline Exact & 0.5 \\
\hline
\end{tabular}

Example 4.2.

$$
\begin{aligned}
I(x, t)= & \frac{1}{2}+\frac{1}{\pi} \int_{0}^{\infty} \exp \left\{-x \zeta\left(1+\zeta^{2}\right)^{-1 / 4} \sin \left(\frac{\tan ^{-1} \zeta}{2}\right)\right\} \\
& \times \sin \left\{\zeta t-\zeta x\left(1+\zeta^{2}\right)^{-1 / 4} \cos \left(\frac{\tan ^{-1} \zeta}{2}\right)\right\} \frac{d \zeta}{\zeta}, \quad x, t \geqslant 0 .
\end{aligned}
$$

$I(x, t)$ is an integral representation for the solution to a problem of wave propagation in a viscoelastic medium, Longman [7], for which approximate solutions have been obtained by Longman [5], [6] by using approximate Laplace transform inversion through rational approximations, and accurate approximations have been obtained by Levin [3] by using a method to accelerate the convergence of Bromwich's integral for Laplace transform inversion, which has been shown to be a special case of the $D$-transformations of Levin and Sidi [4].

The integrand of the integral on the right-hand side of (4.1) is not of the form which suits our purposes as a simple analysis shows. However, by making the change of variables $\zeta=z^{2}$, we can put the integral in the form $\int_{0}^{\infty} f(z) d z$, where

$$
f(z)=e^{\phi(z)} \sin (\theta(z)) h(z),
$$

where $\phi \in A^{(1)}$ with $\bar{\phi}(z)=x z / \sqrt{2}, \theta \in A^{(2)}$ with $\bar{\theta}(z)=t z^{2}-x z / \sqrt{2}$, and $h(z)=$ $2 / z$ hence $h \in A^{(-1)}$, i.e., $k=1, m=2, \gamma=-1$. Therefore, $\sigma=-1$. Now we compute the zeros of $\sin (\bar{\theta}(z))$ by solving the quadratic equation $t z^{2}-x z / \sqrt{2}=q \pi, q$ integer. We considered the cases for which $x=1$, and $t=0.1,0.5,1,100$. It is easy to see that $x_{q}$ is the positive solution of the quadratic equation above for $q=0,1, \ldots$. Taking then $\psi(z)=e^{\bar{\phi}(z)} \cos (\bar{\theta}(z)) / z^{2}$, and using the $W$-transformation with the $x_{l}$ as determined above, we obtain the results given in Table 4.2. The results for $x=1$, $t=0.1,0.5,1.0$, are much more accurate than those given by Levin [3], although they have been obtained with much less labor than those of Levin.

Example 4.3.

$$
\begin{aligned}
I= & \int_{0}^{\infty} \sin \left(\frac{a^{2}}{t^{2}}\right) \cos \left(b^{2} t^{2}\right) \frac{d t}{t^{2}}=\frac{\sqrt{\pi}}{4 \sqrt{2} a} \\
& \times\left[\sin (2 a b)+\cos (2 a b)+e^{-2 a b}\right], \quad a>0, b>0 .
\end{aligned}
$$




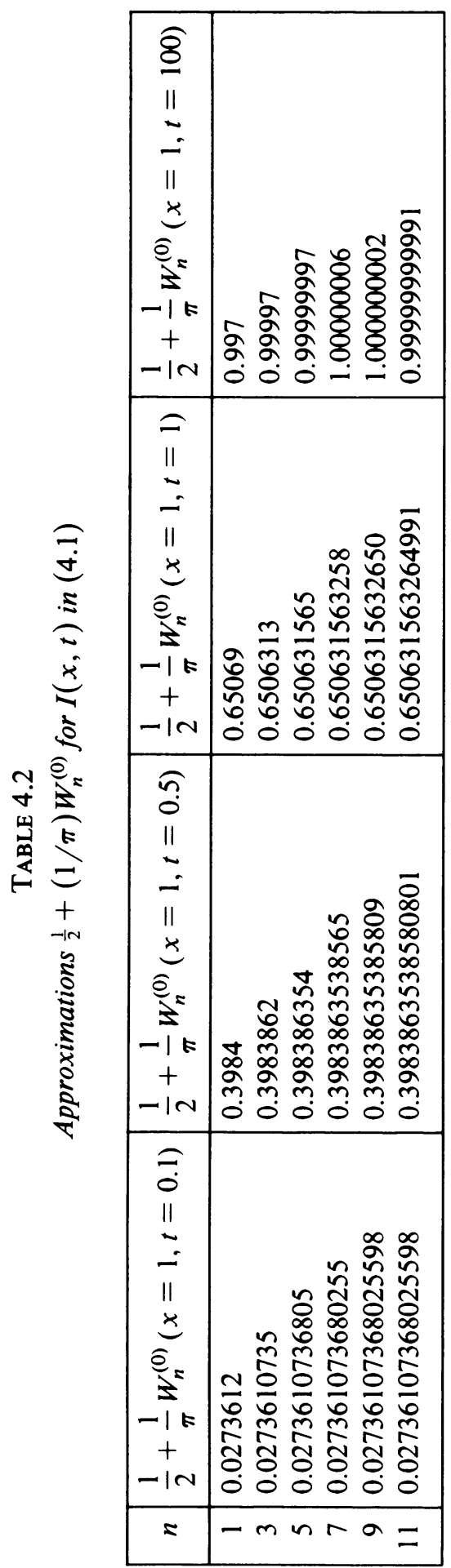


The integrand in this example has an infinite number of oscillations both as $t \rightarrow \infty$ and as $t \rightarrow 0$. Therefore we divide the range of integration into two: $(0, T)$ and $(T, \infty)$. We then map the interval $(0, T)$ to $(1, \infty)$ by the change of variable $\tau=T / t$, hence obtaining two infinite integrals whose integrands oscillate at infinity an infinite number of times. For this example we take $a=\sqrt{\pi}, b=\sqrt{\pi} / 2$ and $T=1$. With this choice of $a, b$, and $T$ we have

$$
I=\int_{1}^{\infty} f(t) d t+\int_{1}^{\infty} f_{1}(t) d t
$$

where

$$
f(x)=\sin \left(\frac{\pi}{x^{2}}\right) \cos \left(\frac{\pi x^{2}}{4}\right) x^{-2}, \quad f_{1}(x)=\sin \left(\pi x^{2}\right) \cos \left(\frac{\pi}{4 x^{2}}\right),
$$

and both $f(x)$ and $f_{1}(x)$ are very oscillatory at $x=\infty$.

Let us denote the $W$-approximations for the integrals $\int_{1}^{\infty} f(t) d t$ and $\int_{1}^{\infty} f_{1}(t) d t$ by $W_{n}^{(j)}[f]$ and $W_{n}^{(j)}\left[f_{1}\right]$, respectively. In Table 4.3 we give the approximations to $I$ obtained as $W_{n}^{(0)}[f]+W_{n}^{(0)}\left[f_{1}\right]$.

TABLE 4.3

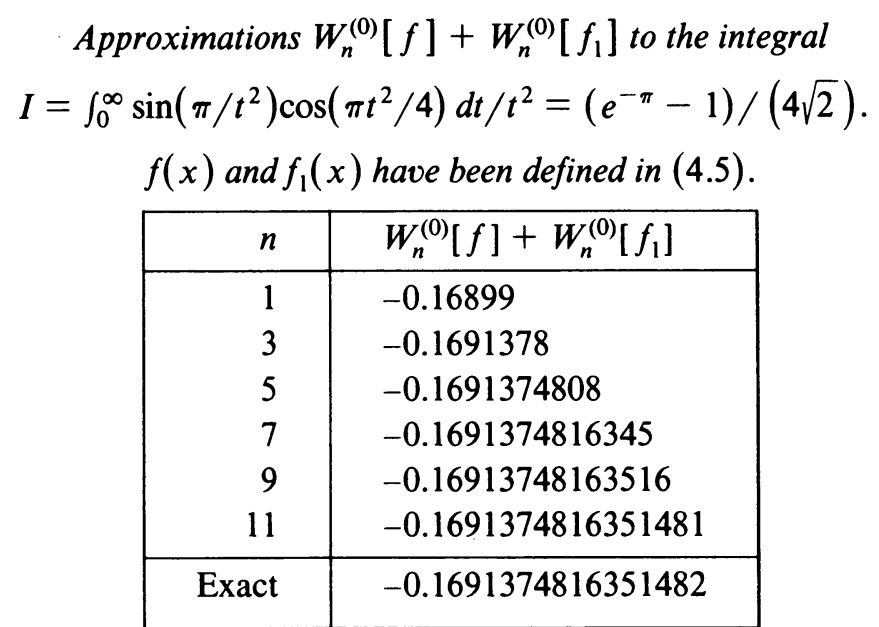

Example 4.4.

$$
I=\int_{0}^{\infty} J_{0}\left(\frac{t^{4}+2 t^{2}+5}{t^{2}+4}\right) \sqrt{t^{2}+9 t+20} d t .
$$

The exact value of this integral is not known to the author. This integral can be dealt with by making use of the remarks at the end of Section 2 .

First of all we have

$$
\theta(x)=\left(x^{4}+2 x^{2}+5\right) /\left(x^{2}+4\right),
$$

$\phi(x)$ constant, and $h(x)=\sqrt{x^{2}+9 x+20}$ in (2.20). Therefore, $\bar{\theta}(x)=x^{2}$, i.e., $m=2, k=0$, and $\gamma=1$. Consequently, the integrand is of the form

$$
f(x)=\cos \left(x^{2}\right) h_{1}(x)+\sin \left(x^{2}\right) h_{2}(x),
$$

where $h_{1}, h_{2} \in A^{(0)}$. Letting $x_{l}=\sqrt{(l+1) \pi}, l=0,1, \ldots$, we have $\psi(x)=$ $\cos \left(x^{2}\right) / x$, hence $\psi\left(x_{l}\right)=(-1)^{l+1} / x_{l}, l=0,1, \ldots$ In Table 4.4 we give some of the results obtained by applying the $W$-algorithm to the integral $I$. 
TABLE 4.4

Approximations $W_{n}^{(0)}$ for the integral

$$
\int_{0}^{\infty} J_{0}\left(\left(t^{4}+2 t^{2}+5\right) /\left(t^{2}+4\right)\right) \sqrt{t^{2}+9 t+20} d t .
$$

\begin{tabular}{|r|l|}
\hline$n$ & \multicolumn{1}{|c|}{$W_{n}^{(0)}$} \\
\hline 1 & 2.61 \\
3 & 2.6273 \\
5 & 2.627159 \\
7 & 2.627160408 \\
9 & 2.62716040106 \\
11 & 2.627160401844 \\
\hline
\end{tabular}

\section{Convergence Properties.}

TheOREM 5.1. Let $f(x), \psi(x)$, and $x_{l}, l=0,1, \ldots$, be as in Section 3. Then for Process I

$$
\left|I[f]-W_{n}^{(j)}\right|=o\left(x_{j}^{-n-1}\right) \text { as } j \rightarrow \infty
$$

and for Process II

$$
\left|I[f]-W_{n}^{(j)}\right|=o\left(n^{-\mu}\right) \quad \text { as } n \rightarrow \infty, \text { for any } \mu>0 .
$$

For a proof of this result, see Sidi [12, Section 6]. We note that the inequality (3.5) plays an important role in the proof of this result.

Recalling that $x_{j}=O\left(j^{1 / m}\right)$ as $j \rightarrow \infty,(5.1)$ can be reexpressed as

$$
\left|I[f]-W_{n}^{(j)}\right|=o\left(j^{-(n+1) / m}\right) \quad \text { as } j \rightarrow \infty,
$$

and this indicates that Process I is inferior to Process II, which, at least numerically, is true.

In [12] it is actually proved that

$$
\left|I[f]-W_{n}^{(j)}\right| \leqslant \max _{j \leqslant l \leqslant j+n+1}\left|\psi\left(x_{l}\right)\right| \max _{j \leqslant l \leqslant j+n+1}\left|\beta\left(x_{l}\right)-\pi_{n}\left(x_{j} / x_{l}\right)\right|,
$$

where $\pi_{n}(\xi)=\sum_{i=0}^{n} \alpha_{n, i} \xi^{i}$ is the best polynomial approximation of degree $n$ to $\beta\left(x_{j} / \xi\right)$ on $[0,1]$. Equation (5.2) follows from (5.3) by using the fact $\psi(x)=O(1)$ for $x \geqslant x_{0}$, and that $\beta\left(x_{j} / \xi\right)$ is infinitely differentiable for $0 \leqslant \xi \leqslant 1$, hence $\mid \beta\left(x_{j} / \xi\right)-$ $\pi_{n}(\xi) \mid=o\left(n^{-\mu}\right)$ as $n \rightarrow \infty$ for any $\mu>0$. (This is a standard result in approximation theory.) Starting from (5.3), the general results given in (5.2) can be sharpened in some cases, as shown below.

Let us go back to Example 4.1. Consider the function

$$
S(x)=\int_{x}^{\infty} e^{i \pi t^{2} / 2} d t=\sqrt{\frac{2}{\pi}} \int_{z}^{\infty} e^{i \tau^{2}} d \tau=\frac{e^{i z^{2}}}{z} b(z),
$$

where $z=x \sqrt{\pi / 2}$ and $b \in A^{(0)}$, but $b(z)$ is not analytic at $z=\infty$. Letting $\zeta=\alpha / z$ for some $\alpha>0$, we map the interval $\alpha \leqslant z \leqslant \infty$ to $0 \leqslant \zeta \leqslant 1$. Then $b(\alpha / \zeta)$ can be expanded as $b(\alpha / \zeta)=\sum_{k=0}^{\infty} c_{k} T_{k}^{*}(\zeta)$, where $T_{k}^{*}(\zeta)$ are the shifted Chebyshev polynomials. It is shown in Miller [8] that $c_{k}=O\left[\exp \left(-\omega k^{2 / 3}\right)\right]$ as $k \rightarrow \infty$, for some 
$\omega>0$ that depends on $\alpha$. Therefore,

$$
b(\alpha / \zeta)-\sum_{k=0}^{n} c_{k} T_{k}^{*}(\zeta)=O\left[\exp \left(-\omega n^{2 / 3}\right)\right] \quad \text { as } n \rightarrow \infty
$$

If in (5.5) the $n$th partial sum of the Chebyshev series is replaced by the best polynomial approximation of degree $n$ to $b(\alpha / \zeta)$ on $[0,1]$, then the right-hand side of (5.5) stays the same. Hence in Example 4.1

$$
\beta\left(x_{j} / \xi\right)-\pi_{n}(\xi)=O\left[\exp \left(-\omega^{\prime} n^{2 / 3}\right)\right] \text { as } n \rightarrow \infty,
$$

consequently,

$$
\int_{0}^{\infty} \sin \left(\frac{\pi}{2} t^{2}\right) d t-W_{n}^{(j)}=O\left[\exp \left(-\omega^{\prime} n^{2 / 3}\right)\right] \quad \text { as } n \rightarrow \infty
$$

for some $\omega^{\prime}>0$ that depends on $x_{j}$.

The result in (5.7) is a significant improvement upon the general result of Theorem 5.1 in that it gives a much better bound on the rate of convergence of the $W_{n}^{(j)}$ approximations to Example 4.1.

We note that if $\beta(x)$ is analytic for all $x \geqslant x_{0}$ up to and including $x=\infty$, then $\beta\left(x_{j} / \xi\right)-\pi_{n}(\xi)=O\left(e^{-\omega n}\right)$ as $n \rightarrow \infty$ for some $\omega>0$. Hence for these cases the estimate for the rate of convergence is even better, specifically $\int_{a}^{\infty} f(t) d t-$ $W_{n}^{(j)}=O\left(e^{-\omega n}\right)$ as $n \rightarrow \infty$.

Acknowledgement. The author wishes to thank Professor P. Rabinowitz and Dr. J. N. Lyness for their comments on the presentation of the material of this work. The computations for this work were carried out on the IBM-370 computer at the Computation Center of the Technion, Haifa.

Appendix-Construction of $\beta(x)$ in Theorem 2.1. Differentiating (2.7) we obtain

$$
v(x)=-\frac{x^{r} \beta(x)}{1+\left[x^{r} \beta(x)\right]^{\prime}} v^{\prime}(x) .
$$

Comparing (A.1) with (2.3) we can see that

$$
p(x)=-\frac{x^{r} \beta(x)}{1+\left[x^{r} \beta(x)\right]^{\prime}} .
$$

Therefore, $\beta(x)$ satisfies the linear first-order inhomogeneous differential equation

$$
\beta^{\prime}(x)+q(x) \beta(x)=-x^{-r},
$$

where

$$
q(x)=\frac{r}{x}+\frac{1}{p(x)} .
$$

If $r=1$, then $\bar{p} \neq 0$, hence $q(x)$, as $x \rightarrow \infty$, has an asymptotic expansion whose first term is $(1+1 / \bar{p}) x^{-1}$. From $(2.5) \bar{p} \neq-1$, therefore $1+1 / \bar{p} \neq 0$, hence $q \in A^{(-1)}$ and $q \notin A^{(-2)}$. If $r \leqslant 0$, then $q(x)$, as $x \rightarrow \infty$, has an asymptotic expansion whose first term is $\left(1 / p_{0}\right) x^{-r}$, where $p_{0}=\lim _{x \rightarrow \infty} x^{-r} p(x) \neq 0$. Hence $q \in A^{(-r)}$ and $q \notin A^{(-r-1)}$. Thus, we have shown that $q \in A^{(-r)}$ and $q \notin A^{(-r-1)}$ for all $r \leqslant 1$. Therefore, $q(x) \sim \sum_{i=0}^{\infty} q_{i} x^{-r-i}$ as $x \rightarrow \infty$. Let us now substitute the expansion 
$\beta(x) \sim \sum_{i=0}^{\infty} \beta_{i} / x^{i}$ in (A.3). We can easily see that $\beta_{0}=-1 / q_{0}$. The coefficients $\beta_{1}, \beta_{2}, \ldots$, can now be determined by solving the recursion relation obtained from (A.3).

Computer Science Department

Technion-Israel Institute of Technology

Haifa, Israel

1. F. B. Hildebrand, Introduction to Numerical Analysis, McGraw-Hill, New York, 1956.

2. D. LEVIN, "Development of non-linear transformations for improving convergence of sequences," Internat. J. Comput. Math., v. B3, 1973, pp. 371-388.

3. D. Levin, "Numerical inversion of the Laplace transform by accelerating the convergence of Bromwich's integral," J. Comput. Appl. Math., v. 1, 1975, pp. 247-250.

4. D. LEVIN \& A. SIDI, "Two new classes of non-linear transformations for accelerating the convergence of infinite integrals and series," Appl. Math. Comput. (In press.)

5. I. M. Longman, "Numerical Laplace transform inversion of a function arising in viscoelasticity," $J$. Comput. Phys., v. 10, 1972, pp. 224-231.

6. I. M. LONGMAN, "On the generation of rational approximations for Laplace transform inversion with an application to viscoelasticity," SIAM J. Appl. Math., v. 24, 1973, pp. 429-440.

7. I. M. LoNGMAN, private communication, 1979.

8. G. F. Miller, "On the convergence of the Chebyshev series for functions possessing a singularity in the range of representation," SIAM J. Numer. Anal., v. 3, 1966, pp. 390-409.

9. A. SIDI, "Convergence properties of some nonlinear sequence transformations," Math. Comp., v. 33, 1979, pp. 315-326.

10. A. SidI, "Some properties of a generalization of the Richardson extrapolation process," J. Inst. Math. Appl., v. 24, 1979, pp. 327-346.

11. A. SiDI, "Analysis of convergence of the $T$-transformation for power series," Math. Comp., v. 35 , 1980 , pp. 833-850.

12. A. SidI, "Extrapolation methods for oscillatory infinite integrals," J. Inst. Math. Appl., v. 26, 1980, pp. 1-20.

13. A. SIDI, "An algorithm for a special case of a generalization of the Richardson extrapolation process," Numer. Math. (In press.) 